\title{
Häufige Anwendungsfehler bei der Aerosoltherapie
}

Bei chronisch obstruktiven Atemwegserkrankungen gelten die Aerosoltherapien mit Inhalatoren und Verneblungsgeräten als klinisch gleich wirksam. Vor allem ältere Patienten bevorzugen Vernebler, da diese als effektiver wahrgenommen werden. Ursache dieser Sichtweise sind vermutlich mangelnde Technik und Fehler bei der Anwendung von Inhalatoren. A. S. Melani et al. haben daher Charakteristika und Handhabungsfehler von Anwendern beider Techniken geprüft. Respir Med 2012; 106: 668-676

Die Beobachtungsstudie (Querschnittsstudie) fand an 24 Zentren in städtischer und ländlicher Umgebung in Italien statt. Eingeschlossen wurden Patienten über 18 Jahre mit spirometriebestätigten chronisch obstruktiven Atemwegserkrankungen (Asthma oder COPD), die ihre Aerosoltherapie regelmäßig zuhause durchführten und einen geplanten Untersuchungstermin in einem der Zentren hatten. Um den Selektionsbias und Untersuchungsaufwand zu minimieren, sollten die Mediziner nur die jeweils ersten beiden Patienten eines Tages in die Studie aufnehmen. Dabei füllten beide Seiten einen Fragebogen aus. Die Patienten sollten zudem die Anwendung der Inhalationstechniken demonstrieren.

\section{Handhabungsfehler bei Verneblungsgeräten \\ $\checkmark$}

Insgesamt 1527 Patienten, die ausschließlich Inhalatoren nutzten, bildeten die OIU-Gruppe („Only Inhaler Users“). Die 137 Teilnehmer der 2. Gruppe („Nebuliser and Inhaler Users“: NIU) wendeten regelmäßig beide Techniken an. Das Durchschnittsalter lag bei 67,7 Jahre in der NIU-Gruppe und 61,1 Jahre in der OIU-Gruppe $(p<0,0001)$. Die Krankheitsschwere unterschied sich zwischen beiden Gruppen deutlich: mittlerer $\mathrm{FEV}_{1} \%$ pred: 62,3 (NIU-Gruppe) vs. 69,9 (OIUGruppe), mittlerer $\mathrm{FEV}_{1} / \mathrm{VC}: \quad 61,0$ vs. 66,3; p jeweils < 0,0001. Die Patienten der NIU-Gruppe mussten in den 12 Monaten vor der Studie öfter im Krankenhaus behandelt werden, Notaufnahmen und Antibiotikatherapien waren ebenfalls häufiger ( $p$ jeweils <0,001). Über Handhabungsfehler berichteten $49 \%$ der NIU-und $36 \%$ derOIU-Patienten $(p=0,009)$.

Gegenüber den Anwendern beider Inhalationstechniken erhielten die Patienten der OIU-Gruppe zur erstmaligen Inhalatorverschreibung häufiger eine Instruktion (85 vs. $65 \%$ ), mehr Patienten wurde die Anwendung demonstriert (59 vs. $43 \%$ ) und bei Nachfolgeuntersuchungen wurden die Inhalatoren häufiger praktisch geprüft (50 vs. 38\%). Auch nach Berücksichtigung des Alters, Inhalationstechnik und jeder Form der Anwendungsinstruktion blieb in der NIU-Gruppe die Häufigkeit von Handhabungsfehlern deutlich größer $(p=0,03)$.

\section{Fazit}

Patienten mit chronisch obstruktiven Atemwegserkrankungen, die Verneblungsgeräten zur Medikamenteninhalation nutzen, waren in dieser Studie im Schnitt älter, von schwereren Obstruktionen betroffen und mussten häufiger zusätzliche medizinische Hilfe in Anspruch nehmen. Außerdem wurden bei der Anwendung von Verneblern laut Autorenteam mehr Handhabungsfehler gemacht.

Matthias Manych, Berlin
HI-Virus

\section{Rauchen senkt Lebenszeit rapide}

Die negativen Auswirkungen des Tabakkonsums sind bei HIV-Infizierten besonders gravierend. Das Forscherteam um Marie Helleberg von der Kopenhagener Universitätsklinik hat in einer Langzeitstudie nachgewiesen, dass rauchende $\mathrm{Pa}-$ tienten eine deutlich niedrigere Lebenserwartung haben als nicht rauchende HIVInfizierte. Eine 35-jährige HIV-Patientin, die raucht, hat demnach eine Lebenserwartung von 62,6 Jahren. Würde sie nicht rauchen, hätte sie eine deutlich höhere Lebenserwartung von 78,4 Jahren. Die Forscher haben für ihre Studie die Daten von 2921 HIV-Infizierten und 10642 Kontrollpersonen aus Dänemark miteinander verglichen. Die Ergebnisse wurden Ende 2012 in Fachjournal Clinical Infectious Diseases veröffentlicht.

\section{Entwöhnung ratsam $\nabla$}

Der Verlust von Lebensjahren - assoziiert mit dem Rauchen - ist bei HIV-infizierten Personen doppelt so hoch wie bei Nichtrauchern. Zudem errechneten die Forscher, dass die Sterblichkeit von HIV-infizierten Rauchern 3-mal höher ist als die von Personen, welche nicht mit dem HIVirus infiziert sind. Diese Ergebnisse unterstreichen die Bedeutung der Raucherentwöhnung speziell bei HIV-Patienten. „Besorgniserregend ist auch, dass über $60 \%$ der Todesfälle bei HIV-Patienten mit dem Rauchen verbunden sind", sagt Marie Helleberg dazu.

\section{Geringere Regeneration $\nabla$}

Rauchen und HIV-Infektion erzeugen einen kumulativen Effekt. Rauchen ist auch ohne HIV-Infektion ein gesundheitliches Risiko. Das Risiko potenziert sich bei HIV-Infizierten, die rauchen. HIV-Patienten altern durch die Infektion zudem schneller und haben nicht so eine hohe Regenerationsfähigkeit wie Gesunde. Zudem hätten viele der Medikamente für die Betroffenen $\mathrm{Ne}$ benwirkungen, die das Risiko von kardiovaskulären Erkrankungen weiter erhöhen.

Pressetext Nachrichtenagentur GmbH 